\title{
recillunds
}

Revista Científica Mundo de la Investigación y el Conocimiento

Katty Leonela Jaramillo Ospina a ; Julieta América Campi Mayorga ${ }^{\text {b; }}$ Tanya del Roció Sánchez Salazar ${ }^{c}$

Informática y ofimática una herramienta pedagógica

Computer and office automation a pedagogical tool

Revista Científica Mundo de la Investigación y el Conocimiento. Vol. 3 núm.3, septiembre, ISSN: 2588-073X, 2019, pp. 1085-1100

DOI: $\underline{10.26820 / \text { recimundo/3.(3).septiembre.2019.1085-1100 }}$

URL: http://recimundo.com/index.php/es/article/view/560

Código UNESCO: 3304 Tecnología de los Ordenadores

Tipo de Investigación: Artículo de Revisión

Editorial Saberes del Conocimiento

Recibido: 15/05/2019

Aceptado: 23/06/2019

Publicado: 30/09/2019

Correspondencia: klejaos@hotmail.com

a. Magister en Administración de Empresas; Magister en Informática Empresarial; Especialista en Redes de Comunicación de Datos; Ingeniera en Sistemas Administrativos Computarizados; Investigador Independiente; Guayaquil, Ecuador; klejaos@ hotmail.com

b. Magister en Gerencia de Proyectos Educativos y Sociales; Magister en Informática Empresarial; Especialista en Redes de Comunicación de Datos; Licenciada en Ciencias de la Educación Especialización Informática Educativa; Ingeniero Tecnológico en Computación y Ciencias de la Informática; Profesora de Segunda Enseñanza Especialización Informática Educativa; Analista de Sistemas; Investigador Independiente; Guayaquil, Ecuador; jullamerica160474@gmail.com

c. Magister en Docencia y Gerencia en Educación Superior; Diploma Superior en Docencia y Evaluación en la Educación Superior; Licenciada en Ciencias de la Educación en la Especialización de Educación Primaria; Universidad Técnica de Babahoyo; Babahoyo, Ecuador; trsanchezs@utb.edu.ec 


\section{Informática y ofimática una herramienta pedagógica}

Vol. 3, núm. 3., (2019)

Katty Leonela Jaramillo Ospina; Julieta América Campi Mayorga; Tanya del Roció Sánchez Salazar

\section{RESUMEN}

Una herramienta ofimática es una recopilación de programas usados en oficinas que permiten crear, modificar, organizar, escanear, imprimir, etc. archivos y documentos. Son ampliamente usados en paquetes, las suites dominantes del mercado es Microsoft Office, es un software pagado, posee sus propios formatos cerrados de documentos para cada uno de sus programas. Como software libre está OpenOffice, son poco adaptables a los cambios que hacen otra suite por tener código abierto. La ofimática difiere de la informática tradicional que trata cada caso con tratamiento distintivo, es decir, se pueden estandarizar las formas y el proceder pero no el contenido del mismo. Las herramientas ofimáticas más comúnmente conocidas son: WORD, EXCEL, POWER POINT Y OUTLOOK. La tecnología de la información no es función exclusiva de las grandes empresas y organizaciones, también las pequeñas empresas (Pymes) deben incluirlas en su funcionamiento diario. En el mercado existen aplicaciones generales a precios accesibles, incluso gratuitos al igual que profesionales capacitados para desarrollar sistemas a la medida y propósito de las empresas. El subestimar esta dinámica global ocasiona a cualquier empresa grande o pequeña, limitaciones en su productividad y crecimiento, mermas de inventarios, perdida de dinero por falta de control, pérdida de clientes al no recibir un servicio de calidad y oportuno, incertidumbre en la toma de decisiones. Algunas tendencias que marcarán el rumbo de la ofimática en el futuro: ampliación de funcionalidades, en la nube, interconexión, accesibilidad y uso. La metodología usada es descriptiva, con un enfoque documental, es decir, revisar fuentes disponibles en la red, como google académico, con contenido oportuno, actualizado y relevante desde el punto de vista científico que enriquezca el análisis del tema planteado en este artículo.

Palabras Claves: Ofimática; Productividad; Eficiencia; Interconexión; Inversión en Capital Humano; Software; Office; Aplicaciones. 


\title{
Informática y ofimática una herramienta pedagógica
}

Vol. 3, núm. 3., (2019)

Katty Leonela Jaramillo Ospina; Julieta América Campi Mayorga; Tanya del Roció Sánchez

Salazar

\begin{abstract}
An office tool is a collection of programs used in offices that allow creating, modifying, organizing, scanning, printing, etc. files and documents. They are widely used in packages, the dominant suites of the market is Microsoft Office, it is a paid software, it has its own closed document formats for each of its programs. As free software is OpenOffice, they are not very adaptable to changes made by another suite because they have open source. Office automation differs from traditional computing that deals with each case with distinctive treatment, that is, the forms and procedures can be standardized, but not the content of the same. The most commonly known office tools are: WORD, EXCEL, POWER POINT AND OUTLOOK. Information technology is not the exclusive function of large companies and organizations, but also small companies (SMEs) must include them in their daily operations. In the market there are general applications at accessible prices, even free as well as trained professionals to develop customized systems and business purpose. Underestimating this global dynamic causes any large or small company, limitations in its productivity and growth, inventory losses, loss of money due to lack of control, loss of customers due to not receiving a quality and timely service, uncertainty in the taking of decisions. Some trends that will mark the course of office automation in the future: expansion of functionalities, in the cloud, interconnection, accessibility and use. The methodology used is descriptive, with a documentary approach, that is, to review sources available on the web, such as academic google, with timely, updated and relevant content from a scientific point of view that enriches the analysis of the topic raised in this article.
\end{abstract}

Key Words: Office Automation; Productivity; Efficiency; Interconnection; Investment in Human Capital; Software; Office; Applications. 


\section{Informática y ofimática una herramienta pedagógica}

Vol. 3, núm. 3., (2019)

Katty Leonela Jaramillo Ospina; Julieta América Campi Mayorga; Tanya del Roció Sánchez Salazar

\section{Introducción.}

Hace algunos años, en las empresas se redactaban las cartas a mano, lo que implicaba el uso de mucho tiempo en esa tarea, una carta para cada cliente o proveedor, si había un error, se tenía que realizar nuevamente. Actualmente, gracias al uso de programas informáticos, se tienen plantillas y modelos que pueden desarrollarse en menos tiempo y mayor cantidad, lo que reduce costes yeleva la productividad.

Ofimática es un acrónimo derivado de los términos oficina e informática cuyo objetivo es simplificar tareas. Abarca todas las herramientas y métodos que se aplican a las actividades de oficina que posibilitan el procesamiento computarizado de datos escritos, visuales y sonoros. No se limita a capturar documentos escritos sino que permite realizar las siguientes actividades:

- Intercambio de información.

- Gestión de documentos administrativos.

- Tratamientos de datos numéricos.

- Planificación de reuniones y administración de cronogramas de trabajo.

El contexto laboral en las últimas décadas se ha caracterizado por el cambio continuo, afectado por la globalización que genera nuevas formas de empleo, flexibilidad laboral e institucional y las innovaciones tecnológicas que impactan las condiciones del mercado de trabajo y a los profesionales. Las nuevas tecnologías han tenido un lugar fundamental en la productividad y crecimiento económico de los países más prósperos. 


\section{Informática y ofimática una herramienta pedagógica}

Vol. 3, núm. 3., (2019)

Katty Leonela Jaramillo Ospina; Julieta América Campi Mayorga; Tanya del Roció Sánchez

Salazar

La nueva generación de niños, creciendo en medio de la tecnología facilita la mirada de las aplicaciones dentro de la cotidianidad, incluso un profesional actualizado en ofimática puede desempeñar el trabajo de varias personas en forma eficiente sin que ello implique explotación del trabajador, todo lo contrario, su capacitación y destreza en el aprovechamiento de las aplicaciones le permitirán dar respuestas oportunas, elevando su productividad y eficiencia.

\section{Metodología.}

Esta investigación está dirigida al estudio de la ofimática una mirada hacia el futuro. Para realizarlo se usó una metodología descriptiva, con un enfoque documental, es decir, revisar fuentes disponibles en la red, como google académico, con contenido oportuno y relevante desde el punto de vista científico para dar respuesta a lo tratado en el presente artículo y que sirvan de inspiración para realizar otros proyectos. Las fuentes consultadas pueden ser estudiadas al final, en la bibliografía.

\section{Resultados.}

Una herramienta ofimática es una recopilación de programas usados en oficinas que permiten crear, modificar, organizar, escanear, imprimir, etc. archivos y documentos. Son ampliamente usados en paquetes, las suites dominantes del mercado es Microsoft Office, es un software pagado, posee sus propios formatos cerrados de documentos para cada uno de sus programas. Como software libre está OpenOffice, son poco adaptables a los cambios que hacen otra suite por tener código abierto. 


\section{Informática y ofimática una herramienta pedagógica}

Vol. 3, núm. 3., (2019)

Katty Leonela Jaramillo Ospina; Julieta América Campi Mayorga; Tanya del Roció Sánchez Salazar

La ofimática no se trata del uso del ordenador individual, promueve la reingeniería de los procesos y sus etapas, usando la informática como instrumento de eliminar, reducir, agilizar los mismos. Difiere de la informática tradicional que trata cada caso con tratamiento distintivo, es decir, se pueden estandarizar las formas y el proceder pero no el contenido del mismo, se trasciende el Modelo cliente-servidor tradicional(Allendez, Junio 2009).

En el entorno ofimático no son necesarios grades equipos, sino que es suficiente ordenadores personales sencillos, conectados entre sí y a internet, formando redes entre los que se comparte información y se abaratan costos. Se ha convertido en una herramienta que crece y se usa cada vez más.

Se define la ofimática como la utilización de ordenadores en la oficina como soporte a los trabajadores de la información que no son especialistas en ordenadores. Se llama ofimática al equipamiento hardware y software usados para idear y crear, coleccionar, almacenar, manipular y transmitir digitalmente la información necesaria en una oficina para realizar tareas y objetivos básicos. La ofimática ayuda a optimizar o automatizar las tareas típicas en una oficina existente (Menéndez, 2009).

La ofimática debe su desarrollo a la aparición de la computadora personal y a su posterior evolución. Con anterioridad las computadoras estaban limitadas al ámbito de la investigación o negocios que pudieran asumir una inversión cuantiosa en materia tecnológica. Con la aparición de la computadora personal se abrió un mundo que habilita la posibilidad de llevar adelante tareas complejas a un bajo costo. En la siguiente tabla se muestra en forma resumida este proceso. 


\section{Informática y ofimática una herramienta pedagógica}

Vol. 3, núm. 3., (2019)

Katty Leonela Jaramillo Ospina; Julieta América Campi Mayorga; Tanya del Roció Sánchez

Salazar

Tabla $\mathbf{N}^{\circ} 1$. Evolución histórica de la ofimática

\begin{tabular}{|c|c|c|}
\hline Año & Fase & Características \\
\hline 1975 - 1980 & Primera & $\begin{array}{l}\text { Se componía de elementos aislados. } \\
\text { No tenían interrelación entre sí. } \\
\text { Sustentada por grandes ordenadores } \\
\text { corporativos. } \\
\text { La formación era muy costosa. }\end{array}$ \\
\hline 1980 - 1990 & Segunda & $\begin{array}{l}\text { Irrumpen los paquetes integrados, si necesitas } \\
\text { uno o dos, sólo se puede adquirir el paquete } \\
\text { completo. } \\
\text { La formación era imprescindible y costosa. } \\
\text { Se abarata la producción de equipos, son más } \\
\text { accesibles al usuario. }\end{array}$ \\
\hline 1990 & Tercera & $\begin{array}{l}\text { Se pueden adquirir solo las aplicaciones que } \\
\text { necesitas. } \\
\text { PC's con altas capacidades en procesos, } \\
\text { monitores en color y tecnologías de } \\
\text { programación orientadas a objetos. } \\
\text { Se reduce el tiempo de formación del usuario al } \\
\text { mínimo y surge el "trabajo en grupo". } \\
\text { Surge la oficina sin papel gracias a las redes. } \\
\text { Surgen las suites como conjunto de } \\
\text { aplicaciones disponibles como productos } \\
\text { independientes. } \\
\text { Todas las aplicaciones comparten datos en } \\
\text { forma transparente. }\end{array}$ \\
\hline
\end{tabular}

Fuente: Elaboración propia

Como consecuencia de la incorporación de la computadora a la oficina, dio pie al desarrollo de distintas aplicaciones especializadas cuyo objetivo era realizar trabajos comunes en forma eficiente. Las planillas de cálculo, los procesadores de textos y motores de base de datos que promueven la mejora continua. 


\section{Informática y ofimática una herramienta pedagógica}

Vol. 3, núm. 3., (2019)

Katty Leonela Jaramillo Ospina; Julieta América Campi Mayorga; Tanya del Roció Sánchez Salazar

Las herramientas ofimáticas más comúnmente conocidas son: WORD, EXCEL, POWER POINT Y OUTLOOK(Grupo Femxa, 2017), se muestra en la figura $\mathrm{N}^{\circ} 1$ las aplicaciones con sus logos como están disponibles en los paquetes o suites de Office:

Figura 1. Aplicaciones Ofimáticas más utilizadas

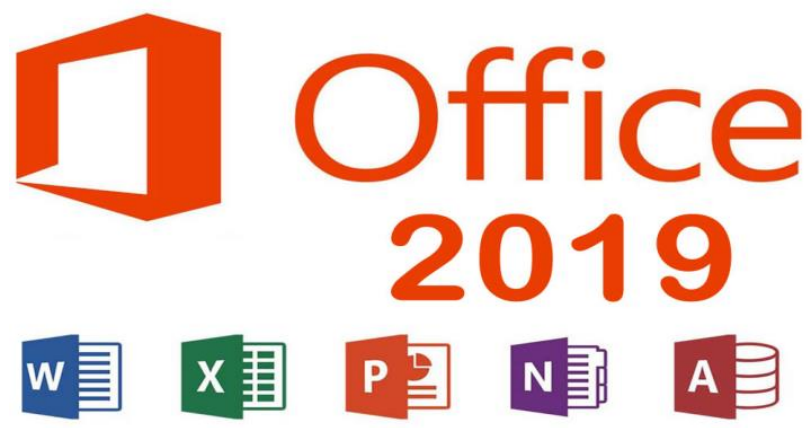

Fuente: Rojas (2018)

Procesador de texto: el WORD es uno de los programas más conocidos de Microsoft Office, permite crear, editar y modificar documentos de textos con formato. Permite trabajar textos con diferentes tipografías (fuentes), incluir imágenes, corrector ortográfico e incluso un diccionario de sinónimos. En sus más recientes actualizaciones, el paquete incluye plantillas para crear documentos concretos como flyers, currículos, portadas para trabajos e índices.

Hojas de cálculo: el útil Excel, permite organizar datos de forma rápida, gracias a las tablas dinámicas se pueden cruzar datos y tener todo en una misma hoja de cálculo. Está compuesto por hojas de cálculo que permite realizar operaciones aritméticas de manera automática, herramientas muy útiles en departamentos de finanzas, presupuestos y contabilidad, entre otros. 


\section{Informática y ofimática una herramienta pedagógica}

Vol. 3, núm. 3., (2019)

Katty Leonela Jaramillo Ospina; Julieta América Campi Mayorga; Tanya del Roció Sánchez

Salazar

Bases de datos: Access es una herramienta muy asertiva en seguimiento de pedidos, proveedores o recopilación de datos. El programa es ideal para almacenar datos, realizar consultas sobre alguna información y realizar informes.

Programa de presentación: Power Point, es excelente para realizar presentaciones visuales, combinar diapositivas multimedia con imagen, sonido, texto e incluso videos.

Programas de correo electrónico: los emails, calendarios para reuniones y directorio de contacto pueden administrarse a través de Outlook. Permite crear cuentas personales y para empresas, crear listas de para hacer envíos, llevar agendas y reuniones con equipos de trabajo.

Microsoft ya tiene disponible la versión 2019 mostrada en la figura $\mathrm{N}^{\circ} 1$ de su suite de escritorio Office 2019 con un incremento en su precio de $10 \%$ con respecto al valor del Office 2016, esta versión es autónoma y no es la versión de Office 365 que se conecta a la Nube. La Office 365 mantuvo su precio igual, sin mostrar variación alguna.

La tecnología de la información no es función exclusiva de las grandes empresas y organizaciones, también las pequeñas empresas (Pymes) deben incluirlas en su funcionamiento diario. En el mercado existen aplicaciones generales a precios accesibles, incluso gratuitos al igual que profesionales capacitados para desarrollar sistemas a la medida y misión de las Pymes.

El subestimar esta dinámica global ocasiona a cualquier empresa grande o pequeña, limitaciones en su productividad y crecimiento, mermas de inventarios, perdida de dinero por falta de control, pérdida de clientes al no recibir un servicio de calidad y oportuno, incertidumbre en la toma de decisiones entre otras razones (Granados, 2017). 


\section{Informática y ofimática una herramienta pedagógica}

Vol. 3, núm. 3., (2019)

Katty Leonela Jaramillo Ospina; Julieta América Campi Mayorga; Tanya del Roció Sánchez Salazar

Los logros y ganancias del paquete de ofimática más conocido en el mundo:Office de Microsoft no solo son ganancias para Redmond, sino que se ha convertido en ejemplo de innovación e integración. Office ya no es solamente Word, Excel y Powerpoint sino una serie de servicios como Yammer, Skype e incluso Sky Drive que lo catapultan como uno de los paquetes más completos e integrados del mercado (Genbeta, 2013).

¿Conviene invertir en capacitación de aplicaciones ofimáticas?

La respuesta siempre será positiva, lo relevante a considerar es quien asume el costo de esta inversión ¿la empresa o el trabajador? Eso dependerá del costo y requerimientos del empleo y la organización. Los cursos y diplomados en esta área ofrecen al participante crear documentos profesionales con Word, el uso y utilidad de la hoja de cálculo o Excel, entre otras aplicaciones.

El manejo de las herramientas ofimáticas aumenta las oportunidades laborales en cualquier campo, incluso existen instituciones educativas cuyos programas de enseñanza están orientados al uso de Tablet y no cuadernos, herramienta clásica de enseñanza tradicional. Facilita el uso y manejo de los dispositivos y potencia el conocer y aprender sus diversas aplicaciones desde muy tierna edad, lo que se traduce en una relación más amistosa y cotidiana con la tecnología y sus aplicaciones.

El mercado de las Tablet ¿una oportunidad?

Las grandes ideas revolucionarias que provocan cambios en industrias o mercados tradicionales se gestan por debajo. La Tablet se está convirtiendo en una versión de computadora personal usada por muchas personas, incluso niños. Un nuevo mercado, una colosal oportunidad 


\section{Informática y ofimática una herramienta pedagógica}

Vol. 3, núm. 3., (2019)

Katty Leonela Jaramillo Ospina; Julieta América Campi Mayorga; Tanya del Roció Sánchez

Salazar

de negocio para empresas que consigan adaptarse a las limitaciones y ventajas de estos dispositivos, ofreciendo paquetes ofimáticos sencillos, que aporten valor o utilidad en diversas áreas: personal, estudios, laboral, etc.

Se inicia un proceso en el que desarrollo de las nuevas tecnologías redefinen el lugar de la niñez, invierte las jerarquías e instala a los niños no sólo como consumidores sino como productores activos de contenido. Por si fuera poco, la conectividad redefine su estatus de pertenencia social, cultural y sus vínculos tanto con pares como con los adultos (Mociulsky, 2018).

Existen detractores del mercado de la Tablet argumentando que son dispositivos de consumo y no de crear contenidos. La Tablet está limitada por las características del dispositivo, pero esto no quiere decir que los procesos de creación no tengan cabida en estos dispositivos de 7 y 10 pulgadas. Apple, Microsoft, Google y Amazon están empujando este nuevo mercado del cual nadie ha proyectado su crecimiento y menos aún cuánto tiempo será. Lo que sí está claro es que la ofimática que se conoce hoy no será la misma dentro de 5 o 10 años.

\section{Tendencias educativas 2018: Robótica y programación}

Actualmente debemos ser conscientes de que vivimos en un entorno completamente tecnificado en muchos aspectos: desde pagar la compra de un artículo mediante un teléfono móvil hasta conseguir amistades y/o pareja en línea. Esta situación ocurre y no te da el tiempo de 


\section{Informática y ofimática una herramienta pedagógica}

Vol. 3, núm. 3., (2019)

Katty Leonela Jaramillo Ospina; Julieta América Campi Mayorga; Tanya del Roció Sánchez Salazar

decidir si quieres ser parte o no, es sencillamente una realidad global en casi todos los aspectos de la rutina diaria.

El impacto de las nuevas tecnologías en la sociedad actual ha sido una herramienta clave para el desarrollo del entorno on line y en éxito de personas en el ámbito laboral que identificaron la ola y surfearon con ella. Lejos de temer el cambio, lo adaptaron y mejoraron a su forma de trabajo, logrando la eficiencia en actividades cotidianas, ahorrando tiempo y dinero.

El uso de la tecnología va más allá de la ofimática, en educación por ejemplo, puede abarcar la creación de contenido, la búsqueda de información o usar internet para visitas virtuales a museos, galerías y otros lugares.

Las actividades de programación en educación está dando un gran éxito, países como Reino Unido, Estonia o Australia los niños de 5 años de edad ya programan y son asignaturas incluidas en los currículos oficiales (Romero, 2018).

Esta área es un eje clave en el futuro cercano debido a la demanda de nuevos empleos y también por todos los beneficios asociados. Algunas razones para apostar por la formación ofimática como prioridad:

- Una buena formación ofimática no aporta solo conocimiento del entorno de las aplicaciones y sus comandos. Aporta la información y destreza en el uso de las funciones y su uso eficiente a la tarea realizada. El Excel por ejemplo es útil en el área de transporte, en recursos humanos o para un gerente que necesite mostrar unos resultados durante una exposición. 


\section{Informática y ofimática una herramienta pedagógica}

Vol. 3, núm. 3., (2019)

Katty Leonela Jaramillo Ospina; Julieta América Campi Mayorga; Tanya del Roció Sánchez

Salazar

- El formador transmite el fruto de su experiencia y aprendizaje formado a lo largo del tiempo, por eso un formador no transmite solamente un ¿Cómo funciona? Sino un ¿Cómo se debe hacer de forma óptima? (González, Marzo de 2003).

Algunas tendencias que marcarán el rumbo de la ofimática en el futuro (Oliveras, 2016):

- Ampliación de funcionalidades: con el paso del tiempo, el entorno digital ha ganado terreno dentro de las funciones del aspecto físico. Las reuniones se realizan por videoconferencias, uso de pantallas táctiles para presentaciones, perfiles más creativos etc. Esta tendencia seguirá imperando en las oficinas, donde la tecnología cada día mostrará más usos.

- En la nube: las herramientas ofimáticas se seguirán desarrollando y perfeccionando para contar con empresas altamente conectadas, con espacios de trabajo permitan el acceso y edición on line de documentos para todos los interesados. Google Drive u Office OneDrive son ejemplo de herramientas en la nube. Según estudio de Gartner, las suites basadas en cloudcomputing representarán el $60 \%$ del sector en la ofimática en 2022 , alcanzando cerca de 700 millones de usuarios (Oliveras, 2016).

- Interconexión: busca no sólo interconexión de los usuarios, sino también entre las distintas herramientas, potenciando la interacción de las empresas a un mismo documento desde diferentes aplicaciones sin modificar el formato de los archivos. 


\section{Informática y ofimática una herramienta pedagógica}

Vol. 3, núm. 3., (2019)

Katty Leonela Jaramillo Ospina; Julieta América Campi Mayorga; Tanya del Roció Sánchez Salazar

- Accesibilidad: el software libre sigue expandiéndose como una alternativa atractiva debido a su bajo o nulo costo versus los programas más costosos. Son revisados constantemente por sus desarrolladores.

- Uso: irán incorporando nuevos sistemas que faciliten su manejo por parte de los usuarios, la confección de diseños más atractivos o la implantación de los comandos de voz.

\section{Conclusiones.}

Una herramienta ofimática es una recopilación de programas usados en oficinas que permiten crear, modificar, organizar, escanear, imprimir, etc. archivos y documentos. Son ampliamente usados en paquetes, las suites dominantes del mercado es Microsoft Office, es un software pagado, posee sus propios formatos cerrados de documentos para cada uno de sus programas. Como software libre está OpenOffice.

Un profesional que domine a la perfección las aplicaciones ofimáticas resultará más productivo para su organización y para sí mismo, porque potenciará sus técnicas en análisis, diseño, organización y presentación de información. La Tablet se está convirtiendo en una versión de computadora personal usada por muchas personas, incluso niños. Un nuevo mercado, una colosal oportunidad de negocio para empresas que consigan adaptarse a las limitaciones y ventajas de estos dispositivos, ofreciendo paquetes ofimáticos sencillos, que aporten valor o utilidad en diversas áreas: personal, estudios, laboral, etc.

En el entorno ofimático no son necesarios equipos de elevado costo. Con ordenadores sencillos conectados entre sí y a internet, formando redes entre los que se comparte información, 


\section{Informática y ofimática una herramienta pedagógica}

Vol. 3, núm. 3., (2019)

Katty Leonela Jaramillo Ospina; Julieta América Campi Mayorga; Tanya del Roció Sánchez

Salazar

se abaratan costos y eleva la productividad y la eficiencia. Las actividades de programación en educación está dando un gran éxito, países como Reino Unido, Estonia o Australia, los niños de 5 años de edad ya programan y son asignaturas incluidas en los currículos oficiales.

Esta área es un eje clave en el futuro cercano debido a la demanda de nuevos empleos y también por todos los beneficios asociados. Algunas razones para apostar por la formación ofimática como prioridad:

- Una buena formación ofimáticaaporta la información y destreza en el uso de las funciones y su uso eficiente a la tarea realizada. El Excel por ejemplo es útil en el área de transporte, en recursos humanos o para un gerente que necesite mostrar unos resultados durante una exposición.

- El formador transmite el fruto de su experiencia y aprendizaje formado a lo largo del tiempo, un formador no transmite solamente un ¿Cómo funciona? sino un ¿Cómo se debe hacer de forma óptima dado los recursos disponibles?

\section{Bibliografía.}

Allendez, P. (Junio 2009). Mercado de trabajo, capacitación continua y trayectoria laboral de los profesionales de Ciencias de la Información. Boletín de la Asociación Andaluz de Bibliotecarios $N^{\circ}$ 94-95, 85-104.

Genbeta. (29 de Enero de 2013). El futuro de la ofimática y su adaptación al mercado de los tablets. Obtenido de Genbeta: www.genbeta.com

González, J. (Marzo de 2003). El futuro de la información: ¿vamos hacia donde queremos? Revista Archipiélago, 55(1).

Granados, G. (Mato de 2017). Ofimática, herramienta para mayor productividad. Obtenido de Visión industrial: www.visionindustrial.com.mx 


\section{Informática y ofimática una herramienta pedagógica}

Vol. 3, núm. 3., (2019)

Katty Leonela Jaramillo Ospina; Julieta América Campi Mayorga; Tanya del Roció Sánchez Salazar

Grupo Femxa. (14 de Abril de 2017). Razones para estudiar ofimática. Obtenido de Emagister: www.emagister.com

Menéndez, R. (15 de Agosto de 2009). Universidad de Murcia. Obtenido de Introducción a la ofimática.: www.um.es

Mociulsky, M. (22 de Mayo de 2018). Consumo digital de niños: Tendencia considerable en Latinoamérica. Obtenido de Quetion Pro: www.questionpro.com

Oliveras, E. (Octubre de 2016). Ofimática y proceso de la información/capital humano. Obtenido de Blog.grupo-pya: www.blog.grupo-pya.com

Romero, M. (03 de Abril de 2018). Tendencias educativas 2018: Robótica y programación. Obtenido de Femxa.es: www.cursofemxa.es 